\title{
Regional Increase in the Expression of the BCAT Proteins in Alzheimer's Disease Brain: Implications in Glutamate Toxicity
}

\author{
Jonathon Hull ${ }^{\mathrm{a}}$, Vinood Patel ${ }^{\mathrm{b}}$, Maya El Hindy ${ }^{\mathrm{a}}$, Christopher Lee ${ }^{\mathrm{a}}$, Esther Odeleye ${ }^{\mathrm{a}}$, \\ Mohammed Hezwani ${ }^{\mathrm{a}}$, Seth Love ${ }^{\mathrm{c}}$, Patrick Kehoe ${ }^{\mathrm{c}}$, Katy Chalmers ${ }^{\mathrm{c}}$ and Myra Conway ${ }^{\mathrm{a}, *}$ \\ ${ }^{a}$ Faculty of Health and Life Sciences, University of the West of England, Bristol, UK \\ ${ }^{\mathrm{b}}$ Faculty of Science and Technology, University of Westminster, London, UK \\ ${ }^{\mathrm{c}}$ Dementia Research Group, Faculty of Medicine and Dentistry, University of Bristol, Bristol, UK
}

Accepted 6 January 2015

\begin{abstract}
.
Background: The human branched chain aminotransferases (hBCATm, mitochondrial and hBCATc, cytosolic) are major contributors to brain glutamate production. This excitatory neurotransmitter is thought to contribute to neurotoxicity in neurodegenerative conditions such as Alzheimer's disease (AD) but the expression of hBCAT in this disease has not previously been investigated.

Objective: The objective of investigating hBCAT expression is to gain insight into potential metabolic pathways that may be dysregulated in AD brain, which would contribute to glutamate toxicity.

Methods: Western blot analysis and immunohistochemistry were used to determine the expression and localization of hBCAT in postmortem frontal and temporal cortex from $\mathrm{AD}$ and matched control brains.

Results: Western blot analysis demonstrated a significant regional increase in hBCATc expression in the hippocampus ( $\uparrow 36 \%$; $p$-values of 0.012), with an increase of $\uparrow 160 \%$ reported for hBCATm in the frontal and temporal cortex ( $p$-values $=4.22 \times 10^{-4}$ and $2.79 \times 10^{-5}$, respectively) in AD relative to matched controls, with evidence of post-translational modifications to hBCATm, more prominent in AD samples. Using immunohistochemistry, a significant increase in immunopositive labelling of hBCATc was observed in the CA1 and CA4 region of the hippocampus ( $p$-values $=0.011$ and 0.026 , respectively) correlating with western blot analysis. Moreover, the level of hBCATm in the frontal and temporal cortex correlated significantly with disease severity, as indicated by Braak staging ( $p$-values $=5.63 \times 10^{-6}$ and $9.29 \times 10^{-5}$, respectively).

Conclusion: The expression of the hBCAT proteins is significantly elevated in AD brain. This may modulate glutamate production and toxicity, and thereby play a role in the pathogenesis of the disease.
\end{abstract}

Keywords: Alzheimer's disease, glutamate metabolism, hBCAT

\section{INTRODUCTION}

In Alzheimer's disease (AD), amyloid- $\beta$ accumulation is thought to initiate a cascade of processes that culminate in synaptic and neuronal damage, the hallmarks of neurodegenerative disease [1]. Although the sequence of events which govern this are not well

*Correspondence to: Myra E. Conway, Faculty of Health and Life Sciences, University of the West of England, Coldharbor Lane, Bristol, BS16 1QY, UK. Tel.: +44 117328 3552; Fax: +44 117328 2904; E-mail: myra.conway@uwe.ac.uk. understood, several metabolic processes are thought to be involved including cellular stress, glutamate toxicity, calcium overload, protein misfolding, and dysregulation of autophagy [2-8]. Under normal physiological conditions, glutamate plays a key role in dendrite and synapse formation and, through glutamatergic transmission, is essential for memory and learning [9]. However, high levels of extracellular glutamate at synapses result in neurotoxicity, thought to be mediated largely through excessive influx of $\mathrm{Ca}^{++}$ [4]. There is also evidence that excitotoxicity leads to 
increased expression of tau protein, neuronal degeneration, and cell death [10-13].

The branched chain aminotransferase (BCAT) proteins are key metabolic proteins responsible for $30 \%$ of de novo glutamate synthesis in rat neurons [14]. They catalyze the reversible transamination of the branched chain amino acids (BCAAs) leucine, isoleucine, and valine to their respective branched chain $\alpha$-ketoacids and glutamate (Scheme 1) [15]. These amino acids can cross the blood-brain barrier and have been demonstrated to act as nitrogen donors for the synthesis of glutamate and glutamine in brain explants and in primary neuronal cultures $[16,17]$. The second step in this metabolic pathway, which is considered the rate limiting step, involves the irreversible oxidative decarboxylation of the branched chain $\alpha$-keto acids into co-enzyme A derivatives; catalyzed by the branchedchain $\alpha$-keto acid dehydrogenase complex (BCKD) [18]. This complex is composed of three catalytic subunits, E1, E2, and E3. Mutations in the genes of the BCKD complex cause maple syrup urine disease (MSUD) [19]. This autosomal recessive disorder of infancy or childhood is characterized by a build-up of both BCAAs and their respective keto-acid derivatives that are neurotoxic to cells, resulting in neurological dysfunction, seizures, and death [19, 20]. Thus, accumulation of products of BCAA metabolism, the $\alpha$-keto acids and glutamate, leads to loss of neuronal integrity that has severe clinical consequences.

BCAA + a-ketoglutarate $\stackrel{\text { hBCAT }}{\rightleftharpoons}$ a-ketoacids + L-glutamate (Scheme 1)

In humans (hBCAT) two isoforms predominate: a ubiquitous mitochondrial isoform, hBCATm (41 $730 \mathrm{Da}$ ), and a largely neuron-specific cytosolic isoform, hBCATc (43 $400 \mathrm{Da}$ ) [21]. Two other variants of hBCATm have also been reported but are not well characterized, the first isolated from placental tissue and the second a splice variant that acts as a corepressor of thyroid hormone nuclear receptors [22, 23 ]. In humans and rodents, hBCATc is predominantly found in the soma and proximal dendrites of glutamatergic and GABAergic neuronal cells, in keeping with its role in contributing to the storage and metabolic pools of glutamate, which is in turn decarboxylated in GABAergic neurons to form GABA [24-26]. Immunohistochemical labelling of hBCATm in rat astrocytes led to the development of the hypothetical model of nitrogen shuttling between neurons and glial cells (Fig. 1) [27-29]. In brief, transamination supports the glutamate/glutamine cycle, which operates between neuronal and astrocytic cells, to compensate for glutamate 'lost' during oxidation in astrocytes. Here, it was proposed that in astrocytes, transamination operates in the direction of glutamate production, whereas the reverse was suggested in neuronal cells, ultimately facilitating the transfer of BCAA nitrogen between the two cell types and providing nitrogen for glutamate/glutamine synthesis (Fig. 1A) [14, 29]. However, in human brain tissue we showed that hBCATm is localized to the brain vasculature rather than astrocytes as observed in rat [26]. As hBCAT is expressed in glutamatergic neurons, we proposed that transamination contributes to glutamate synthesis and the expression of hBCATm in the endothelial cells of the vasculature supports astrocytic regulation of glutamate uptake from the synaptic cleft (Fig. 1B) [26, 30].

Although hBCATs play a pivotal role in brain glutamate metabolism, their possible contribution to diseases associated with glutamate toxicity (thought to be relevant to the pathogenesis of a range of neurodegenerative diseases) has not previously been evaluated $[2,3,9]$. In this study we have investigated the cellular distribution and expression of the hBCATs in postmortem brain tissue from subjects with $\mathrm{AD}$ and matched controls. We provide the first evidence that the $\mathrm{hBCAT}$ proteins show regional upregulation in AD relative to age-matched controls. Not only is upregulation evident but there is also evidence of differential posttranslation modification of $\mathrm{hBCATm}$ in $\mathrm{AD}$, which correlates with Braak stage, indicating a link with disease severity.

\section{MATERIALS AND METHODS}

\section{Materials}

Peroxidase substrate, 3,3'-diaminobenzidine (DAB), Vectashield hard-set fluorescent mountant, goat polyclonal antibody to rabbit IgG, biotin-labelled secondary polyclonal antibody to $\mathrm{IgG}$, and avidin (Vectastain ABC kit) were obtained from Vector labs (Peterborough, UK). Rabbit polyclonal antibodies to hBCATc and hBCATm were purchased from Insight Biotechnology limited (Wembley, UK). Mouse monoclonal antibody to hBCATm was purchased from Abcam (Cambridge, UK). We previously demonstrated the specificity of the hBCATc and hBCATm antibodies for western blotting and immunohistochemistry [26]. Alexafluor 488 and 647 were purchased from Invitrogen (Paisley, UK). Mouse monoclonal antibody to detect S-glutathionylation (Source Bioscience, UK). Chemiluminescent HRP substrate was purchased from 

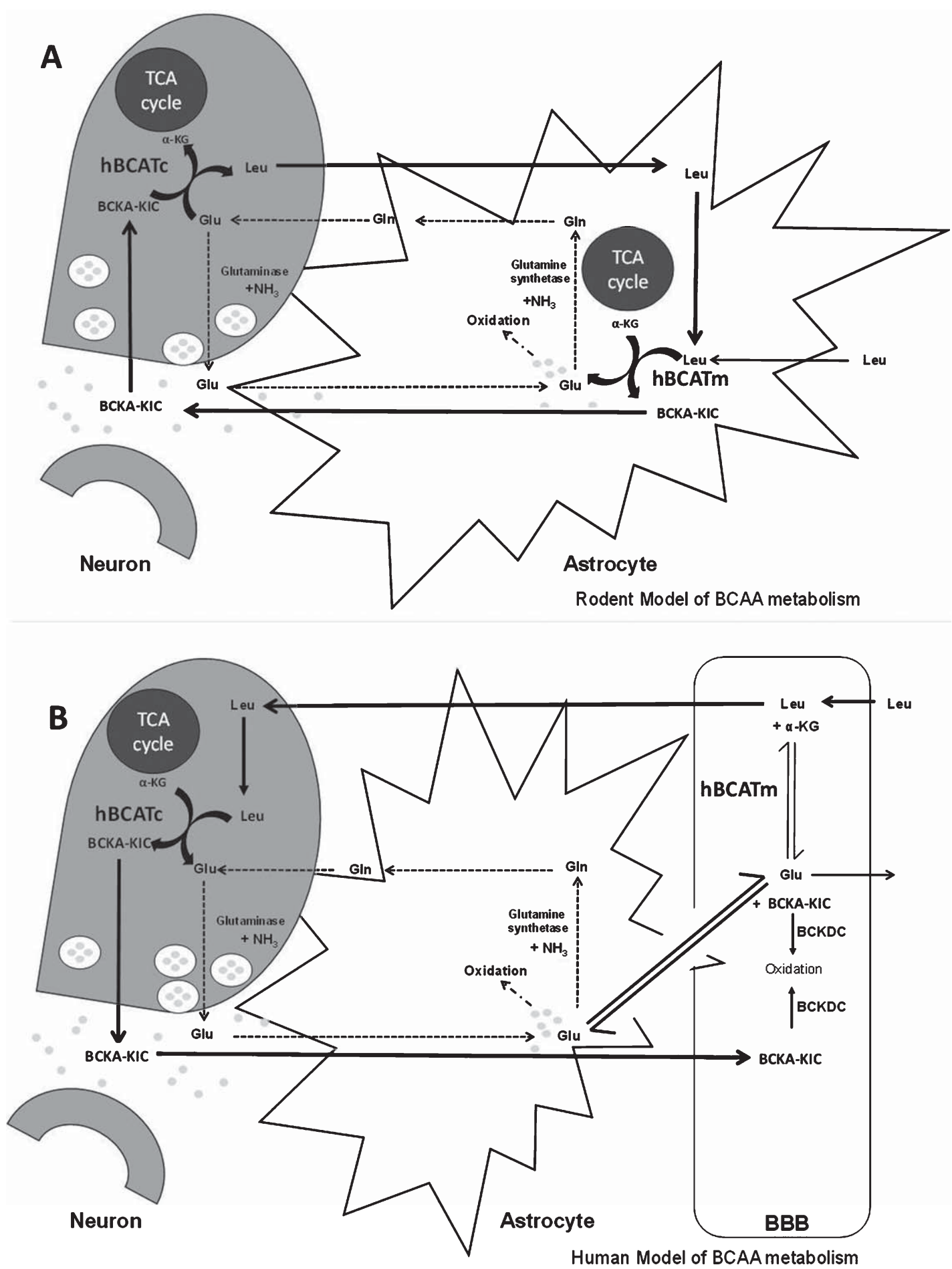

Fig. 1. Branched chain amino acid metabolism. Rodent model: The glutamate/glutamine cycle (dotted lines) operates to sustain neuronal glutamate. However, some glutamate taken up by astrocytes is oxidized and this 'lost' glutamate is replenished by transamination reaction. In astrocytes, transamination operates in the direction of glutamate production, whereas the reverse was suggested in neuronal cells, ultimately facilitating the transfer of BCAA nitrogen between the two cell types and providing nitrogen for glutamate/glutamine synthesis [14, 29]. Human model: In human brain tissue, hBCATm is localized to the endothelial cells of the vasculature [26]. Here, hBCATc transamination is considered to contribute to glutamate production, as hBCATc is expressed in glutamatergic neurons, and the role of hBCATm in the vasculature supports astrocytic regulation of glutamate uptake from the synaptic cleft [26] (reviewed in [30]). 
Millipore (Watford, UK) and mouse monoclonal antibody to glyceraldehyde 3-phosphate dehydrogenase (GAPDH) from Santa Cruz (Canada). All other chemicals were obtained from Fisher Scientific (Loughborough, UK) or Sigma-Aldrich (Dorset, UK).

\section{Brain tissue preparation}

The study was approved by North Somerset and South Bristol Research Ethics Committee, Bristol, UK. All brain tissue used in this study was from brains donated to the South West Dementia Brain Bank at the University of Bristol, UK. For western blot analysis, $40 \mathrm{AD}$ brains and 40 age- and gender-matched controls with both shorter and longer term postmortem delays were assessed (Tables 1, 2). For immunohistochemistry, $30 \mathrm{AD}$ brains and 30 age- and gender-matched controls were studied (Table 3 ). The right cerebral hemisphere, half brainstem and half cerebellum had been fixed in $10 \%$ buffered formalin for 3 weeks before being extensively sampled for paraffin histology and neuropathological assessment by Prof. Seth Love, con- sultant neuropathologist. The left hemibrain had been sliced and frozen at $-80^{\circ} \mathrm{C}$. All AD cases had a diagnosis of definite $\mathrm{AD}$ according to the Consortium to Establish a Registry for AD criteria, and a Braak tangle stage of IV, V, or VI. Controls did not have a history of dementia and did not have any neuropathological abnormalities apart from occasional tangles in the transentorhinal region and hippocampus in a few cases (i.e., all had a Braak tangle stage of III or less). Subject details are summarized in Tables 1-3.

Paraffin sections $7 \mu \mathrm{m}$ in thickness were taken from the right temporal lobe in the coronal plane of the lateral geniculate body. The sections encompassed the hippocampus, parahippocampal, and fusiform gyri. From some brains, sections of the cerebellum were also taken as an internal control as this region does not show evidence of AD pathology apart from occasional diffuse plaques. The sections were subsequently processed for immunohistochemistry as described.

For western blot analysis, $250 \mathrm{mg}$ of tissue from the left temporal, frontal cortex, and hippocampus was collected in RNase free tubes. For protein

Table 1

Alzheimer's disease and control cases used in western blot analysis of hBCATc and hBCATm. All cases chosen had a postmortem (PM) delay of less than $72 \mathrm{~h}$, had no significant comorbidities (such as vascular dementia or Parkinson's disease), and were age- and gender-matched

\begin{tabular}{|c|c|c|c|c|c|c|c|c|c|}
\hline Case & Age & Gender & Braak & PM delay & Case & Age & Gender & Braak & PM delay \\
\hline AD 1 & 95 & M & 4 & 27 & Control 1 & 93 & M & 3 & 38 \\
\hline AD 2 & 89 & $\mathrm{~F}$ & 5 & 39 & Control 2 & 88 & $\mathrm{~F}$ & 0 & 72 \\
\hline AD 3 & 88 & $\mathrm{~F}$ & 6 & 64 & Control 3 & 88 & $\mathrm{~F}$ & 2 & 28 \\
\hline $\mathrm{AD} 4$ & 84 & $\mathrm{~F}$ & 6 & 20.5 & Control 4 & 84 & $\mathrm{~F}$ & 1 & 17 \\
\hline AD 5 & 80 & M & 4 & 24 & Control 5 & 80 & M & 0 & 46 \\
\hline AD 6 & 80 & $\mathrm{~F}$ & 5 & 50 & Control 6 & 80 & $\mathrm{~F}$ & 0 & 72 \\
\hline $\mathrm{AD} 7$ & 69 & $\mathrm{M}$ & 6 & 72 & Control 7 & 69 & M & 2 & 66 \\
\hline AD 8 & 65 & M & 5 & 39 & Control 8 & 64 & M & 0 & 16 \\
\hline AD 9 & 86 & $\mathrm{~F}$ & 5 & 73 & Control 9 & 89 & $\mathrm{~F}$ & 2 & 15 \\
\hline AD 10 & 64 & M & 5 & 67 & Control 10 & 64 & M & 2 & 12 \\
\hline AD 11 & 70 & $\mathrm{~F}$ & 6 & 25 & Control 11 & 70 & M & 2 & 50 \\
\hline AD 12 & 71 & M & 6 & 30 & Control 12 & 71 & M & 1 & 25 \\
\hline AD 13 & 71 & $\mathrm{~F}$ & 5 & 67 & Control 13 & 72 & $\mathrm{~F}$ & 0 & 24 \\
\hline AD 14 & 72 & M & 6 & 65 & Control 14 & 72 & M & 1 & 42 \\
\hline AD 15 & 73 & M & 5 & 17 & Control 15 & 73 & M & 1 & 33 \\
\hline AD 16 & 74 & M & 5 & 48 & Control 16 & 73 & M & 2 & 36 \\
\hline AD 17 & 74 & M & 5 & 50 & Control 17 & 73 & M & 3 & 35 \\
\hline AD 18 & 74 & $\mathrm{~F}$ & 6 & 12 & Control 18 & 73 & $\mathrm{~F}$ & 1 & 59 \\
\hline AD 19 & 74 & M & 5 & 24 & Control 19 & 75 & M & 2 & 48 \\
\hline AD 20 & 74 & M & 4 & 55 & Control 20 & 75 & M & 3 & 6 \\
\hline AD 21 & 91 & M & 3 & 43 & Control 21 & 92 & M & 2 & 34 \\
\hline AD 22 & 93 & M & 6 & 20 & Control 22 & 94 & M & 2 & 40 \\
\hline AD 23 & 83 & $\mathrm{~F}$ & 5 & 5 & Control 23 & 83 & $\mathrm{~F}$ & 2 & 24 \\
\hline AD 24 & 89 & $\mathrm{~F}$ & 5 & 28 & Control 24 & 89 & $\mathrm{~F}$ & 3 & 47 \\
\hline AD 25 & 96 & $\mathrm{~F}$ & 4 & 53 & Control 25 & 94 & $\mathrm{~F}$ & 2 & 21 \\
\hline AD 26 & 85 & M & 6 & 50 & Control 26 & 85 & M & 2 & 31 \\
\hline AD 27 & 76 & M & 5 & 11 & Control 27 & 76 & M & 2 & 23 \\
\hline AD 28 & 78 & M & 6 & 50 & Control 28 & 78 & M & 1 & 48 \\
\hline AD 29 & 78 & M & 5 & 22 & Control 29 & 78 & M & 2 & 12 \\
\hline AD 30 & 61 & M & 5 & 38 & Control 30 & 62 & $\mathrm{M}$ & 0 & 4 \\
\hline Average & 78.57 & $11(\mathrm{~F}): 19(\mathrm{M})$ & 5.13 & 39.62 & & 78.57 & $10(\mathrm{~F}): 20(\mathrm{M})$ & 1.53 & 34.80 \\
\hline
\end{tabular}


Table 2

Alzheimer's disease (AD) and control cases used in western blot analysis of hBCATc and hBCATm. All cases chosen had a postmortem (PM) delay of less than $15 \mathrm{~h}$, had no significant comorbidities (such as vascular dementia or Parkinson's disease) and were age- and gender-matched

\begin{tabular}{|c|c|c|c|c|c|c|c|c|c|}
\hline Case & Age & Gender & Braak & PM delay & Case & Age & Gender & Braak & PM delay \\
\hline AD 31 & 76 & M & 5 & 11 & Control 31 & 75 & M & 3 & 6 \\
\hline AD 32 & 69 & M & 5 & 12 & Control 32 & 77 & M & 3 & 10 \\
\hline $\mathrm{AD} 33$ & 81 & M & 6 & 4 & Control 33 & 81 & M & 2 & 3 \\
\hline AD 34 & 86 & M & 6 & 11.5 & Control 34 & 88 & $\mathrm{~F}$ & 3 & 15 \\
\hline $\mathrm{AD} 35$ & 74 & $\mathrm{~F}$ & 6 & 12 & Control 35 & 74 & $\mathrm{~F}$ & 0 & 12 \\
\hline AD 36 & 83 & $\mathrm{~F}$ & 5 & 5 & Control 36 & 89 & $\mathrm{~F}$ & 2 & 15 \\
\hline AD 37 & 83 & M & 5 & 11 & Control 37 & 82 & M & 2 & 3 \\
\hline AD 38 & 80 & M & 5 & 5 & Control 38 & 78 & M & 2 & 12 \\
\hline AD 39 & 64 & M & 6 & 9 & Control 39 & 64 & M & 2 & 12 \\
\hline AD 40 & 60 & $\mathrm{~F}$ & 6 & 5 & Control 40 & 62 & M & 0 & 4 \\
\hline Average & 75.60 & $3(\mathrm{~F}): 7(\mathrm{M})$ & 5.50 & 8.55 & & 77.00 & $3(\mathrm{~F}): 7(\mathrm{M})$ & 1.90 & 9.20 \\
\hline
\end{tabular}

Table 3

Alzheimer's disease (AD) and control cases used in immunohistochemical analysis of hBCATc and hBCATm. All cases chosen had a postmortem (PM) delay of less than $72 \mathrm{~h}$, had no significant comorbidities (such as vascular dementia or Parkinson's disease) and were age- and gendermatched

\begin{tabular}{|c|c|c|c|c|c|c|c|c|c|}
\hline Case & Age & Sex & Braak & PM delay & Case & Age & Sex & Braak & PM delay \\
\hline$\overline{\mathrm{AD}} 1$ & 85 & $M$ & 6 & 50 & Control 1 & 85 & $\mathrm{M}$ & 2 & 31 \\
\hline AD 2 & 95 & $\mathrm{~F}$ & 5 & 28 & Control 2 & 95 & $\mathrm{~F}$ & 4 & 10 \\
\hline AD 3 & 89 & $\mathrm{~F}$ & 5 & 28 & Control 3 & 89 & $\mathrm{~F}$ & 3 & 47 \\
\hline $\mathrm{AD} 4$ & 86 & $\mathrm{~F}$ & 5 & 72 & Control 4 & 87 & $\mathrm{~F}$ & 3 & 47 \\
\hline AD 5 & 69 & M & 6 & 72 & Control 5 & 69 & M & 2 & 66 \\
\hline AD 6 & 65 & M & 5 & 39 & Control 6 & 64 & M & 0 & 16 \\
\hline AD 7 & 85 & M & 5 & 60 & Control 7 & 86 & M & 3 & 16 \\
\hline AD 8 & 95 & M & 4 & 27 & Control 8 & 94 & M & 2 & 40 \\
\hline AD 9 & 80 & M & 4 & 24 & Control 9 & 80 & M & 0 & 46 \\
\hline AD 10 & 88 & $\mathrm{~F}$ & 6 & 64 & Control 10 & 88 & $\mathrm{~F}$ & 2 & 28 \\
\hline $\mathrm{AD} 11$ & 89 & $\mathrm{~F}$ & 6 & 4 & Control 11 & 89 & $\mathrm{~F}$ & 2 & 15 \\
\hline AD 12 & 80 & $\mathrm{~F}$ & 5 & 51 & Control 12 & 80 & $\mathrm{~F}$ & 0 & 39 \\
\hline AD 13 & 78 & M & 6 & 49 & Control 13 & 77 & M & 1 & 42 \\
\hline AD 14 & 87 & M & 6 & 36 & Control 14 & 87 & M & 2 & 24 \\
\hline AD 15 & 93 & M & 6 & 20 & Control 15 & 93 & M & 3 & 38 \\
\hline AD 16 & 74 & M & 5 & 24 & Control 16 & 73 & M & 3 & 35 \\
\hline AD 17 & 76 & M & 5 & 11 & Control 17 & 76 & M & 2 & 23 \\
\hline AD 18 & 78 & M & 6 & 50 & Control 18 & 78 & M & 1 & 48 \\
\hline AD 19 & 71 & $\mathrm{~F}$ & 5 & 67 & Control 19 & 72 & $\mathrm{~F}$ & 0 & 24 \\
\hline AD 20 & 74 & $\mathrm{~F}$ & 6 & 12 & Control 20 & 73 & $\mathrm{~F}$ & 1 & 59 \\
\hline AD 21 & 93 & $\mathrm{~F}$ & 5 & 60 & Control 21 & 93 & $\mathrm{~F}$ & 1 & 15 \\
\hline $\mathrm{AD} 22$ & 73 & M & 5 & 17 & Control 22 & 73 & M & 1 & 33 \\
\hline $\mathrm{AD} 23$ & 78 & $\mathrm{~F}$ & 6 & 21 & Control 23 & 78 & $\mathrm{~F}$ & 1 & 22 \\
\hline $\mathrm{AD} 24$ & 69 & M & 5 & 12 & Control 24 & 70 & M & 2 & 50 \\
\hline $\mathrm{AD} 25$ & 96 & $\mathrm{~F}$ & 4 & 53 & Control 25 & 94 & $\mathrm{~F}$ & 2 & 21 \\
\hline AD 26 & 79 & M & 6 & 28 & Control 26 & 80 & M & 3 & 67 \\
\hline AD 27 & 78 & M & 5 & 22 & Control 27 & 78 & M & 2 & 12 \\
\hline AD 28 & 88 & M & 5 & 3 & Control 28 & 90 & M & 2 & 45 \\
\hline AD 29 & 71 & M & 6 & 30 & Control 29 & 71 & M & 1 & 25 \\
\hline AD 30 & 83 & $\mathrm{~F}$ & 5 & 5 & Control 30 & 83 & $\mathrm{~F}$ & 2 & 24 \\
\hline Average & 81.50 & $12(\mathrm{~F}): 18(\mathrm{M})$ & 5.30 & 34.63 & & 81.50 & $12(\mathrm{~F}): 18(\mathrm{M})$ & 1.77 & 33.60 \\
\hline
\end{tabular}

homogenization, $1 \mathrm{~mL}$ of protein homogenization buffer (1\% SDS, 0.1 M NaCl, $10 \mathrm{mM}$ Tris $\mathrm{HCl} \mathrm{pH} \mathrm{7.6,}$ $1 \mu \mathrm{M}$ PMSF, and $1 \mathrm{mg} / \mathrm{L}$ aprotinin) was added to each sample and homogenized for $15 \mathrm{~s} \times 2$ using a Precellys 24 lysis homogenizer. The tubes were centrifuged at $13,000 \mathrm{rpm}$ at $4^{\circ} \mathrm{C}$ for $15 \mathrm{~min}$. Following centrifugation, $50 \mu \mathrm{L}$ aliquots of the supernatant were prepared and assayed for protein concentration using the Brad- ford method. These samples were subsequently stored at $-80^{\circ} \mathrm{C}$ before western blot analysis.

\section{Immunofluorescence}

Sections were placed in a $60^{\circ} \mathrm{C}$ oven overnight prior to immunohistochemistry and immunofluorescence to aid adhesion. The sections were subsequently 
dewaxed in clearene $(2 \times 5 \mathrm{~min})$ and dehydrated in $100 \%$ ethanol $(2 \times 3 \mathrm{~min})$. Following dehydration, sections were pre-treated using EDTA ( $1 \mathrm{mM}$ EDTA, $0.05 \%$ Tween $20, \mathrm{pH} 8.0$ ) prior to blocking with horse serum (1/10 dilution in PBS). Sections were incubated for $20 \mathrm{~h}$ with primary antibody ( $1 / 50$ for hBCATc and 1/100 for MAP2). Subsequently, the slides were washed in PBS $(2 \times 3 \mathrm{~min})$ and incubated in species specific secondary antibody (1/1000 for Alexafluor 488 and 647) in the dark for $60 \mathrm{~min}$. The slides were washed in deionized water $(2 \times 5 \mathrm{~min})$ and mounted in aqueous mountant. Images were acquired using Andor IQ software (Cairn Research Ltd, Faversham, UK) and a confocal Nikon Eclipse 80i microscope.

\section{Immunohistochemistry}

Initial section treatment occurred in an identical manner to immunofluorescence. After dehydration, endogenous peroxidase was quenched in $0.9 \%$ hydrogen peroxide/methanol solution for $30 \mathrm{~min}$ at room temperature. Standard immunohistochemistry was performed as per Vectastain ABC protocol (with citrate pre-treatment and $20 \mathrm{~h}$ primary antibody incubation) prior to immersion in copper sulphate $\left(16 \mathrm{mM} \mathrm{CuSO} 4.5 \mathrm{H}_{2} \mathrm{O}, 0.123 \mathrm{M} \mathrm{NaCl}\right)$ for $4 \mathrm{~min}$ and counterstaining with Harris's hematoxylin $(25 \%$ Gill hematoxylin). The slides were dehydrated in $100 \%$ ethanol $(2 \times 5 \mathrm{~min})$, cleared in $100 \%$ clearene $(2 \times 3 \mathrm{~min})$, and mounted in clearium. Sections were viewed, scored, and imaged on a Nikon Eclipse 50i and 80 i microscope.

\section{Scoring protocol}

For anatomical reference, labelling was scored in the same region of each section: the collateral sulci of the temporal lobe and the overlying cortex and areas CA1 and CA4 of the hippocampus. The sections were examined under a $20 \times$ objective and the degree of labelling designated on an arbitrary semi-quantitative scale: $0=$ no labelling, $1=$ weak labelling of occasional cells $(<10$ cells), $2=$ labelling of many cells $(15-30$ cells), $3=$ strong labelling of many cells (30+ cells). Labelling was scored according to cell type: neuronal for hBCATc and vascular for hBCATm.

For histometric image analysis, slides were imaged using a Nikon 80i and analyzed using NIS imaging software for the area of image labelled and mean pixel density, to give an integrated density. This was performed on the $\mathrm{CA} 4$ region at $\times 10$ magnification.

\section{Overexpression of $h B C A T$ proteins}

The overexpression and purification of the hBCAT proteins are described in Davoodi et al. [30]. In brief, using E. coli BL21DE3 cells transformed with pET28a, N-terminal $6 \times$ histidine tagged hBCAT-cDNA vectors, hBCAT proteins were overexpressed following induction with $1 \mathrm{M}$ IPTG. Following extraction and affinity purification using nickel-NTA agarose (Qiagen Ltd, West Sussex), the N-terminal $6 \times$ histidine affinity tag was cleaved by (100 NIH units) thrombin. Final purification of each hBCAT isoform was obtained using a HiTrapTM Q HP strong anion column (GE Healthcare, Buckinghamshire). The purified hBCAT proteins were dialyzed at $4^{\circ} \mathrm{C}$ overnight into storage buffer $(50 \mathrm{mM}$ Tris $\mathrm{pH} 7.4,150 \mathrm{mM} \mathrm{NaCl}$, $5 \mathrm{mM}$ glucose, $1 \mathrm{mM}$ EDTA, $1 \mathrm{mM} \alpha$-ketoisocaproate (KIC), $5 \mathrm{mM}$ DTT) and stored at $-20^{\circ} \mathrm{C}$ (in $30 \%$ $\mathrm{w} / \mathrm{v}$ glycerol). The concentration of purified protein was determined using the Schaffner and Weissmann method [31], where the estimated yield was $10 \mathrm{mg}$ of purified protein/L of E. coli. Using 12\% SDS-PAGE and quadruple time of flight mass spectrometry purity of each isoform was determined to be $>98 \%$.

\section{Western blot analysis}

Aliquots of overexpressed protein were calculated to each standard concentration using the Schaffner and Weissmann method [31] and in NuPAGE ${ }^{\circledR}$ LDS sample buffer $(4 \times)$ and loaded on to a NuPAGE ${ }^{\circledR}$ Novex $^{\circledR}$ 4-12\% Bis-Tris $1 \mathrm{~mm}$ gel. Proteins were separated with $1 \times$ NuPAGE $^{\circledR}$ MES SDS Running Buffer for $1 \mathrm{~h}$ at 200 volts. For subject samples, $20 \mu \mathrm{g}$ of protein from each supernatant was used. The gels were prepared for immunoblotting using the SNAP ID system (Millipore). The membranes were blocked with $0.5 \%$ non-fat milk powder in TBST $(0.05 \%$ Tween 20 in Tris buffered saline $-0.02 \mathrm{M}$ Tris, $0.5 \mathrm{M} \mathrm{NaCl}$, $\mathrm{pH}$ 7.5). Primary antibody (1/1000 for hBCATc and GAPDH, and 1/1500 for hBCATm) was prepared in $0.5 \%$ non-fat milk powder in TBST and added for 10 min prior to several washes with TBST. Secondary antibody (1/5000) linked with HRP, was added for 10 min and then washed with TBST. The positive bands were visualized using chemiluminescent HRP substrate. GBX developer and fixer were used for 10 and $20 \mathrm{~s}$ respectively. Integrated area densitometry was carried out with using Image $\mathrm{J}^{\mathrm{TM}}$. These results were measured in relation to the GAPDH loading control. For re-probing, membranes were incubated with 0.5 $\mathrm{M} \mathrm{NaOH}(7 \mathrm{~min})$ and washed in TBST $(3 \times 10 \mathrm{~min})$. 
The membrane was subsequently treated from blocking step.

\section{RESULTS}

Regional increase of $h B C A T c$ expression in $A D$ brain relative to matched controls

Immunohistochemistry and western blot analysis was used to evaluate the differential expression of hBCATc in the hippocampus, frontal, and temporal cortex of AD brain relative to matched controls (Figs. 2 and 3). Using histometric semi-quantitative scoring, a significant increase $(p=0.026)$ in hBCATc expression was observed, with intense staining of neurons (25/30 cases) in the $\mathrm{CA} 4$ region of $\mathrm{AD}$ relative to matched controls brains (Fig. 2A-2D). Labelling of hBCATc was restricted to neurons as illustrated using the neuronal specific marker, microtubule-associated protein 2 (Supplementary Figure 1) [26]. A significant increase $(p=0.011)$ in immunopositive neuronal cells for hBCATc in the CA1 region of the hippocampus was also reported (Fig. 2E-H). Western blot analysis supported these findings, which confirmed that there was a significant increase in hBCATc in the hippocampus of AD relative to matched controls (Fig. 2I, J). The densitometry of hBCATc was calculated relative to the corresponding GAPDH density, which showed no alteration between $\mathrm{AD}$ and control brains (Supplementary Figure 2). Moreover, to evaluate the impact of postmortem delay on hBCAT expression, we assessed the expression of both hBCAT proteins
Control
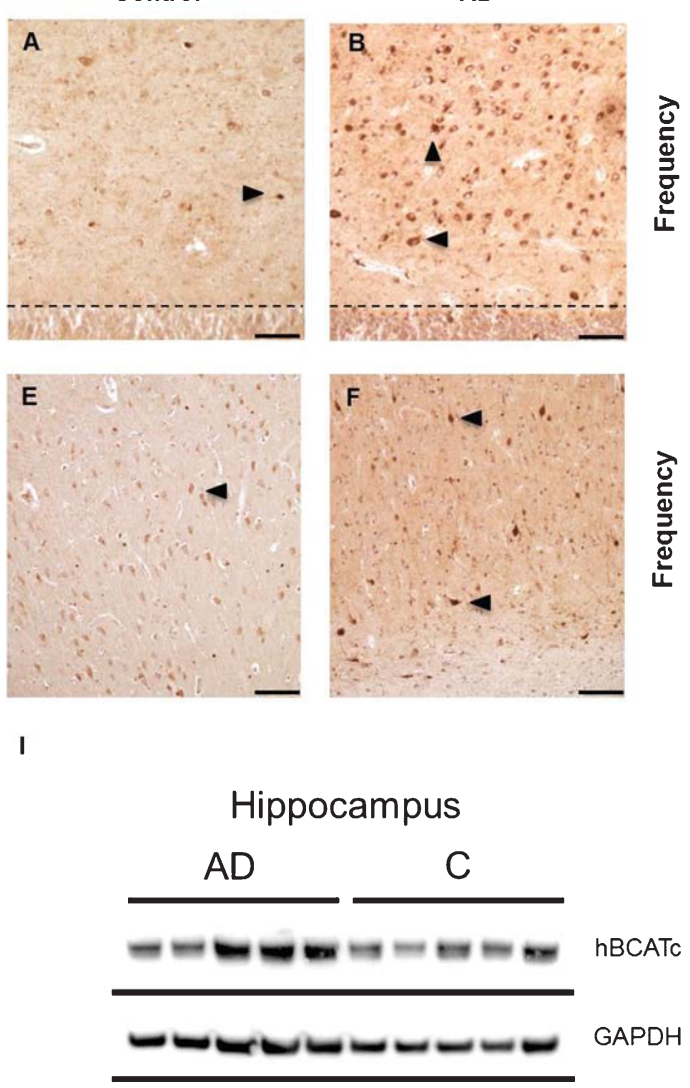

AD
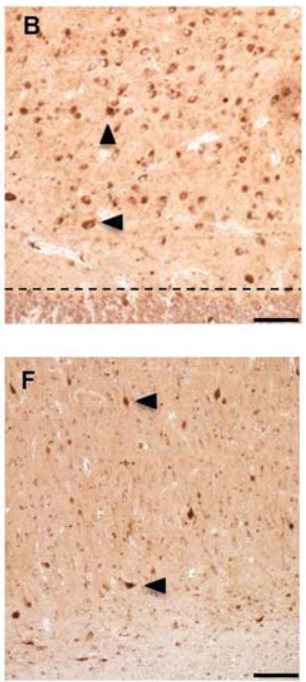

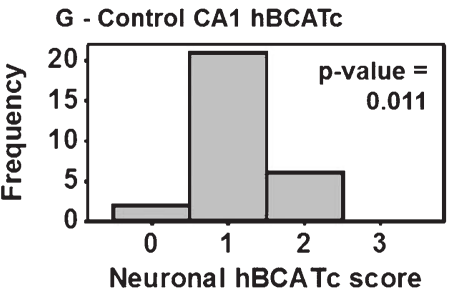

J - Hippocampus hBCATc

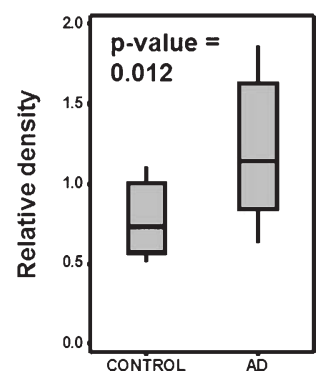

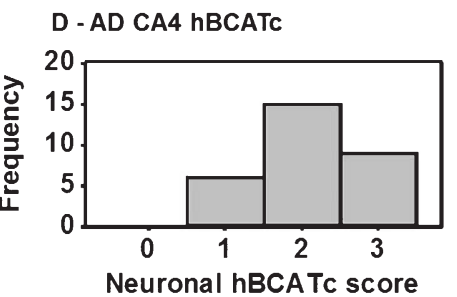

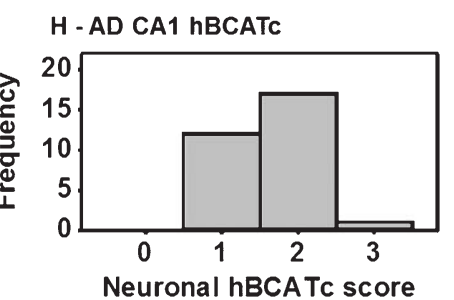

Fig. 2. Increased hBCATc expression in the hippocampus of AD brains. CA4 region of the hippocampus in a control (A) and AD brain (B) showing intensely labelled neurons (large arrows) and the granule cell layer (dotted line). CA1 region of the hippocampus in a control (E) and $\mathrm{AD}(\mathrm{F})$ subject showing intensely labelled neurons (large arrows). C, D, G, H) The slides were scored on a 0-3 scoring system and analyzed for significance using the Wilcoxon-Mann-Whitney test in Minitab ${ }^{\mathrm{TM}}$ as described in materials and methods. I) Western blot analysis of hippocampal tissue. The density of each band was measured using Image ${ }^{\mathrm{TM}}$ software (Wayne Rasband, National Institute of Health, USA) and analyzed for significance using a one-way ANOVA test in Minitab ${ }^{\mathrm{TM}}$. J) Interquartile range (box) sample variability (whiskers) and the median (horizontal line within the interquartile range) are shown. Magnification for A, B, E, and F, $\times 10$. Scale bar: $200 \mu \mathrm{m}$. 

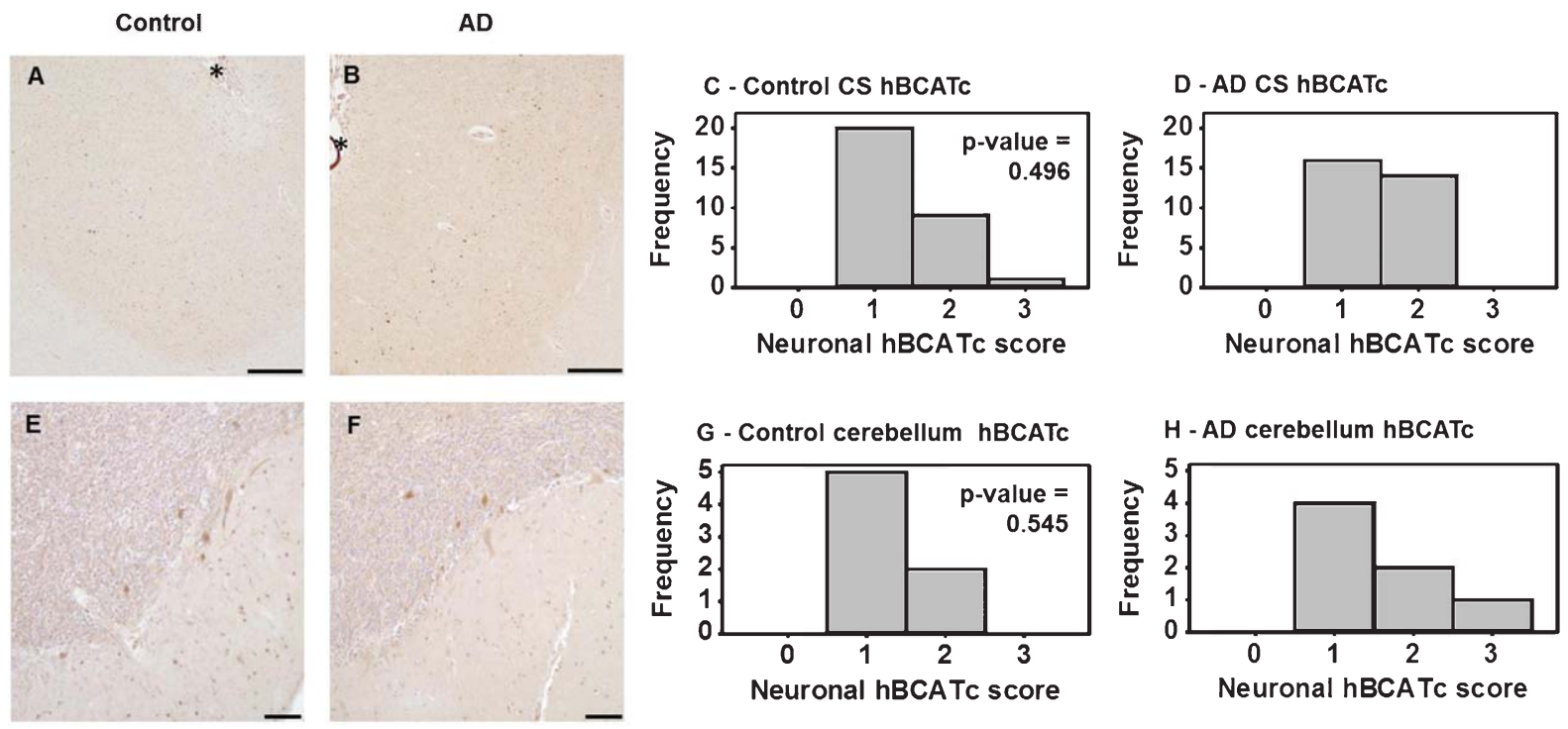

I

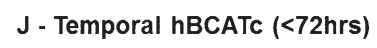

K - Temporal hBCATc (<15hrs)

\section{Temporal}
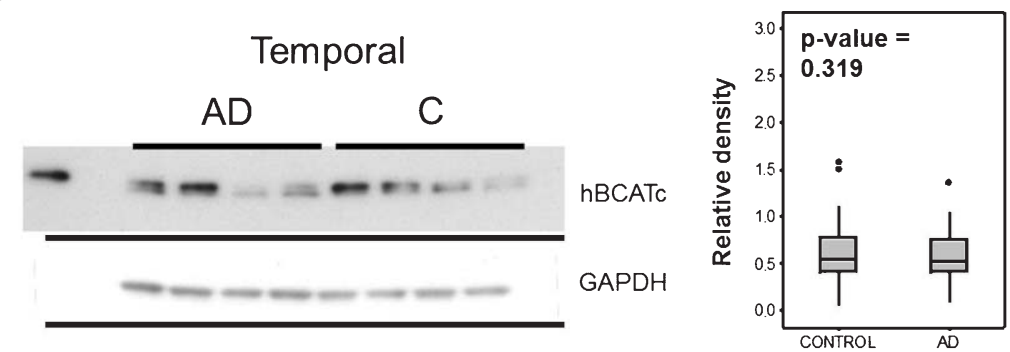

$\mathbf{L}$

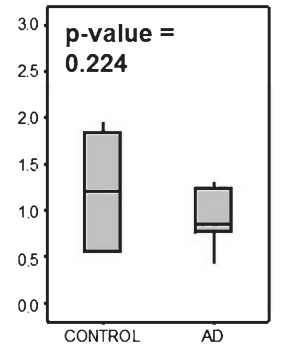

N - Frontal hBCATc $(<15 \mathrm{hrs})$
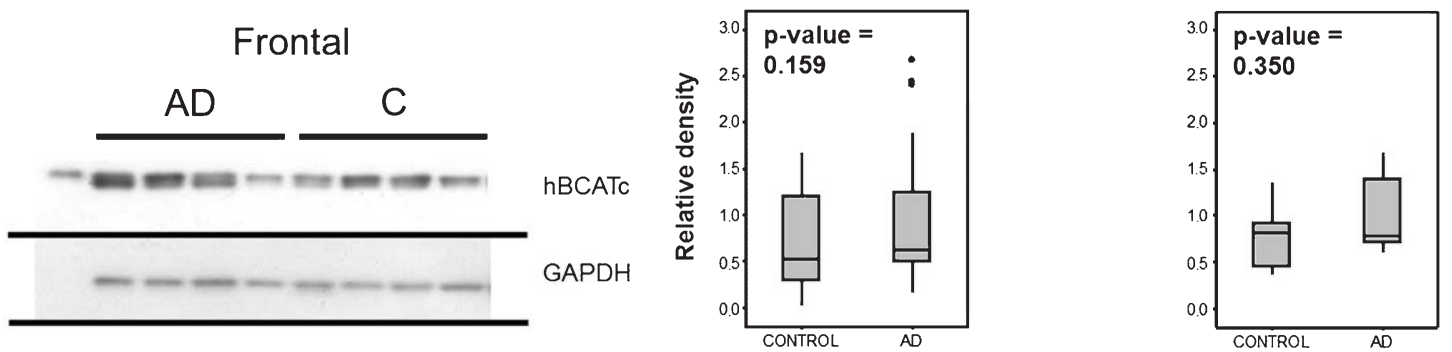

Fig. 3. Immunohistochemistry and western blot analysis of hBCATc in the frontal and temporal cortex of AD and control brains. Collateral sulci $\left(\mathrm{CS}^{*}\right)$ region of the temporal cortex in a control (A) and AD brain (B). Molecular and nuclear layer of the cerebellum in a control (E) and AD (F) brain. C, D, G, H) The slides were scored on a 0-3 scoring system and analyzed for significance using the Wilcoxon-Mann-Whitney test in Minitab ${ }^{\mathrm{TM}}$. Western blot analysis of temporal cortex homogenate (I) and the frontal cortex (L). The density of each band was measured using Image $\mathrm{J}^{\mathrm{TM}}$ software (Wayne Rasband, National Institute of Health, USA) and analyzed for significance using a two-way ANOVA test in Minitab $^{\mathrm{TM}}$. J, K, M, N) Interquartile range (box) sample variability (whiskers) and the median (horizontal line within the interquartile range). Magnification for A, B, E, and F, X10. Scale bar: $200 \mu \mathrm{m}$.

over time, where no change was observed, indicating that postmortem delay does not impact the expression of these proteins (Supplementary Figure 3).

Although positive staining was reported throughout the frontal and temporal regions, it was not as intense as observed in the hippocampal region and there was no significant increase in staining in the collateral sulci region (Fig. 3A-D, $p=0.496$ ). As expected, there was also no difference in labelling for hBCATc in the cerebellum of $\mathrm{AD}$ subjects relative to matched controls, an area not affected by AD pathology (Fig. 3E-H, $p=0.545)$. Using a two-way ANOVA analysis of 
Control
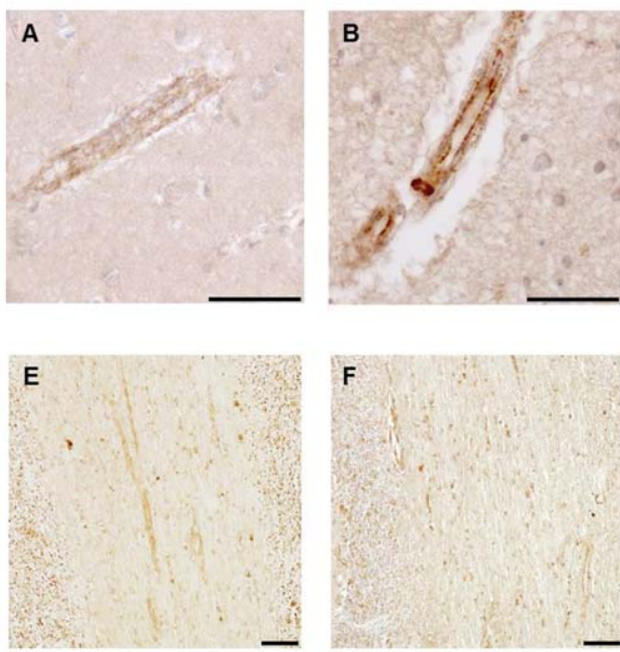

1

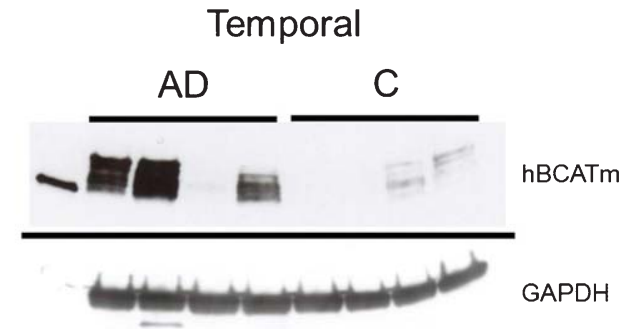

L

Frontal

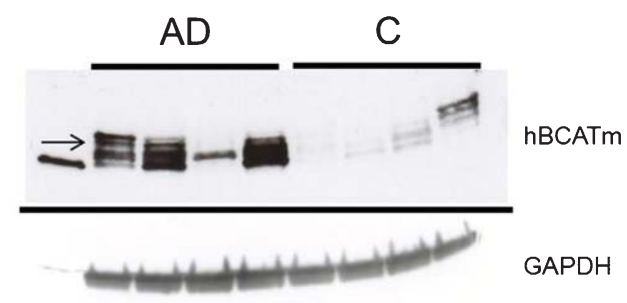

C - Control temporal hBCATm

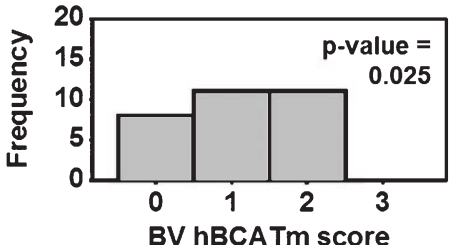

BV hBCATm score

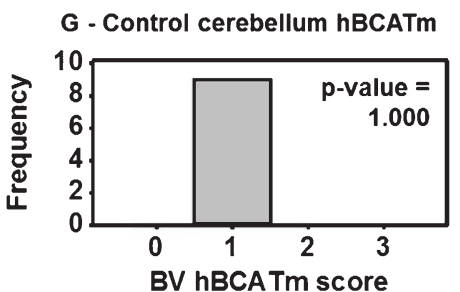

$\mathrm{J}$ - Temporal hBCATm (<72hrs)

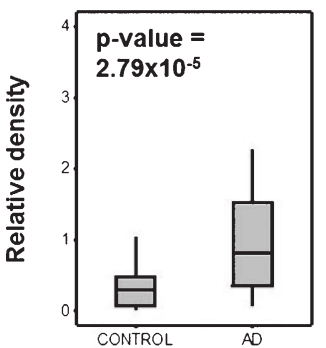

M - Frontal hBCATm (<72hrs)

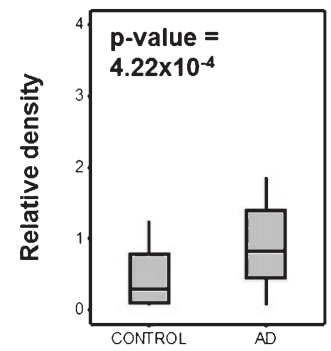

D - AD temporal hBCATm

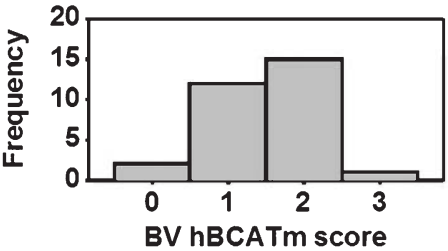

H - AD cerebellum hBCATm

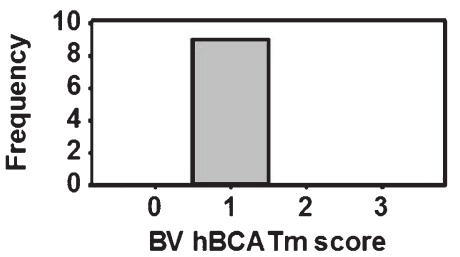

K - Temporal hBCATm (<15hrs)

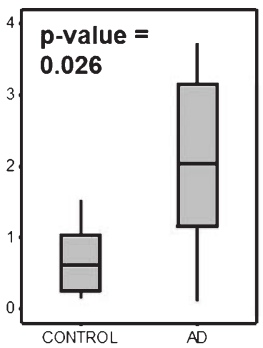

$\mathrm{N}$ - Frontal hBCATm (<15hrs)

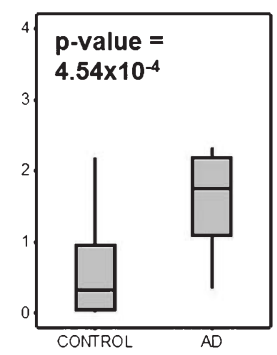

Fig. 4. Widespread increase in hBCAT expression in AD brain. Vessels of the temporal cortex in a control (A) and AD brain (B). White matter of the cerebellum in a control (E) and $\mathrm{AD}(\mathrm{F})$ brain. $\mathrm{C}, \mathrm{D}, \mathrm{G}, \mathrm{H})$ The slides were scored on a $0-3$ scoring system and analyzed for significance using the Wilcoxon-Mann-Whitney test in Minitab ${ }^{\mathrm{TM}}$. Western blot analysis of hBCATm in temporal cortex homogenate (I) and frontal cortex (L). The density of each band was measured using Image ${ }^{\mathrm{TM}}$ software (Wayne Rasband, National Institute of Health, USA) and analyzed for significance using a two-way ANOVA test in Minitab ${ }^{\mathrm{TM}} . \mathrm{J}, \mathrm{K}, \mathrm{M}, \mathrm{N}$ ) Interquartile range (box) sample variability (whiskers) and the median (horizontal line within the interquartile range) are shown. Magnification for A and B, X40; for E and F, X10. Scale bar: A and B, $50 \mu \mathrm{m}$; E and F, $200 \mu \mathrm{m}$.

western blot data, these observations were upheld, where western blot data correlated with histometric analysis (Fig. 3I-N and Supplementary Figure 4, respectively). These studies indicate that there is a regional increase in hBCATc expression, the area most affected by AD pathology.
Increased hBCATm expression in AD brain was more widespread than for $h B C A T c$

As previously described, labelling of hBCATm was largely restricted to blood vessels, throughout all brain areas (Fig. 4A-H) [26]. Here, a significant increase 

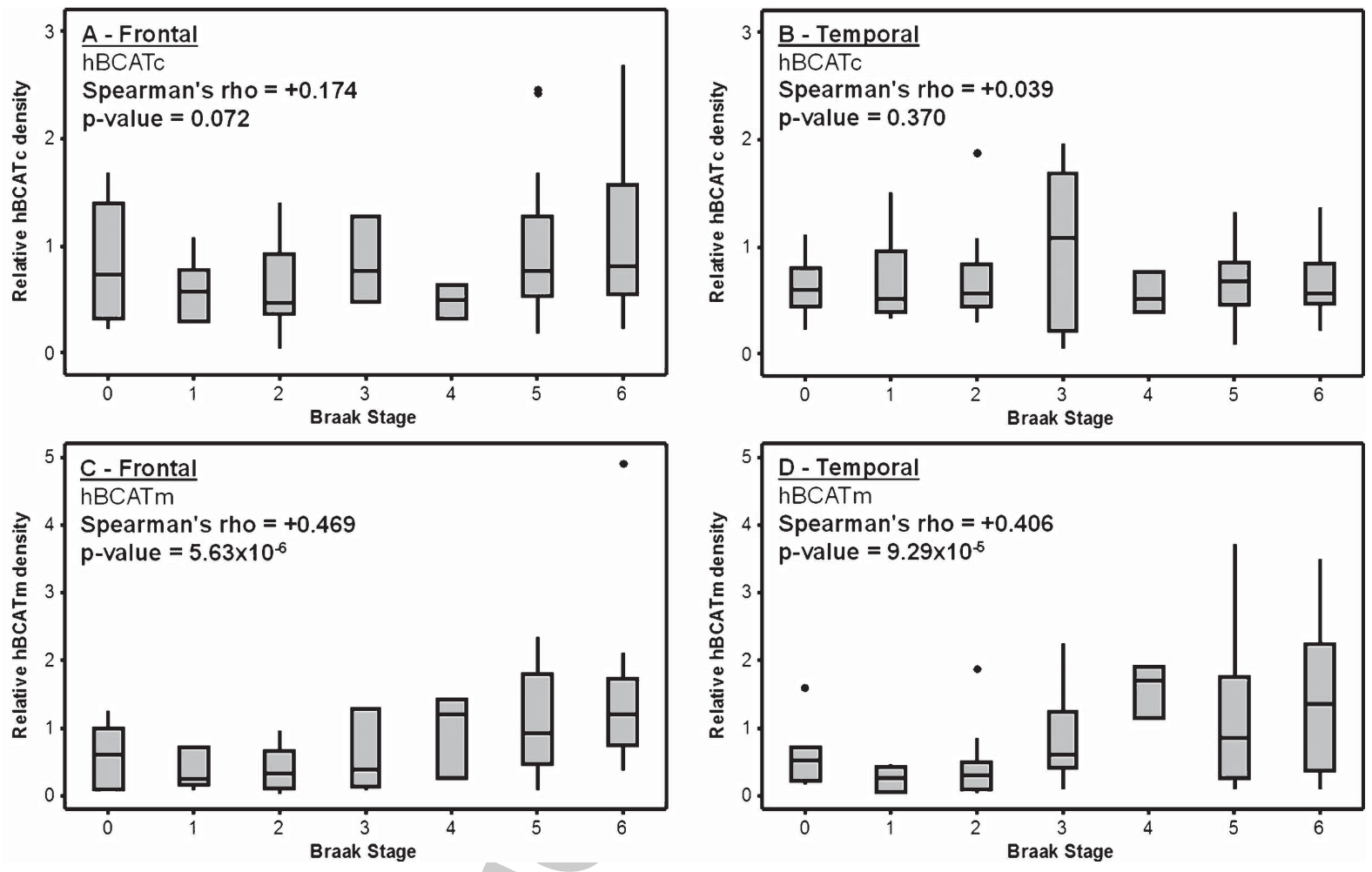

Fig. 5. Box and whisker plots of hBCAT expression correlated with Braak stage. A, B) Box and whisker plots of hBCATc. C, D) Box and whisker plots of hBCATm. Protein expression was estimated relative to GAPDH using densitometry analysis as described in materials and methods and correlated with Braak stage. Panels show interquartile range (box) sample variability (whiskers) and the median (horizontal line within the interquartile range).

in hBCATm staining $(p=0.025)$ was observed in the temporal cortex of $\mathrm{AD}$ brain relative to matched controls, which correlated with western blot analysis $\left(p=2.79 \times 10^{-5}\right)($ Fig. $4 \mathrm{~A}-\mathrm{D}$ and I-J, respectively) . Increased hBCATm expression was also observed in the CA1 and CA4 of the hippocampal region (Supplementary Figure 5). Similar to that reported for hBCATc, there was no increase in hBCATm expression in the cerebellum (Fig. 4E-H, $p=1.0$ ). Two-way ANOVA analysis of hBCATm expression in the frontal cortex also showed a significant increase in expression (Fig. 4L-M, $p=4.22 \times 10^{-4}$ ). Analysis of brain samples with $\mathrm{a} \leq 15 \mathrm{~h}$ postmortem delay also showed a significant increase in hBCATm expression in the temporal and frontal cortex (Fig. $4 \mathrm{~K}$ and N, respectively).

\section{Increased hBCATm expression correlated with Braak stage}

Box and whisker plots were used to evaluate if increased hBCAT expression correlated with Braak stage or gender. No correlation was observed between hBCATc protein density and Braak stage (Fig. 5A, $\mathrm{B})$. However, as observed in Fig. 5C and D there is a positive correlation between $\mathrm{hBCATm}$ relative protein density and the corresponding Braak stage for each patient in the frontal and temporal region. Here, a trend is observed showing an increase in Braak stage, especially at stages 4-6 when compared to $0-3$ as hBCATm density increases, and this is reflected in the Spearman's rank correlation of +0.469 (frontal, $p=5.63 \times 10^{-6}$ ) and 0.406 (temporal, $\left.p=9.29 \times 10^{-5}\right)$.

A two-way ANOVA did not show any significant differences between sex for both hBCATc and hBCATm in frontal and temporal regions (Fig. 6). However, as expected in the frontal region, male and female $\mathrm{AD}$ patients had a significantly higher hBCATm expression compared to male controls (Fig. 6C, $p=0.008$ and 0.004 , respectively) and a similar pattern was observed in the temporal region (Fig. 6D, $p=0.004$ and 0.008 , respectively). 

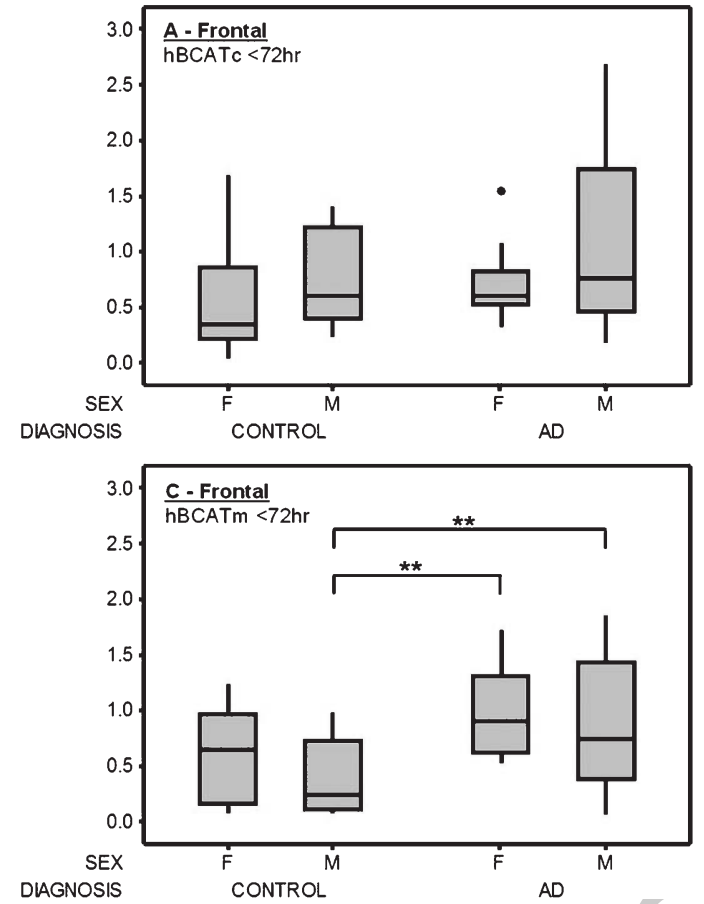
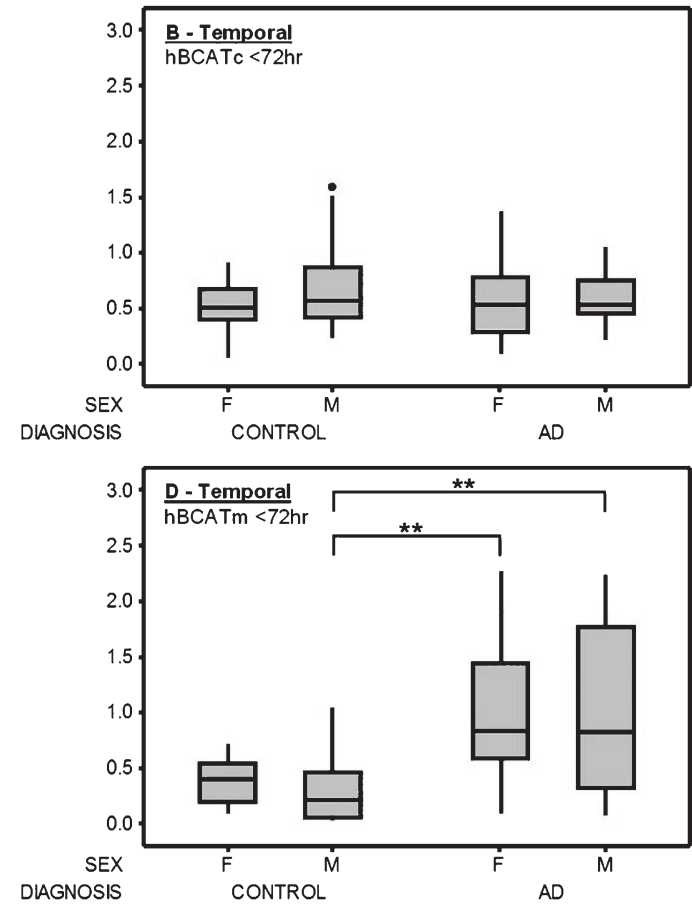

Fig. 6. Tukey Post-hoc analysis of two-way ANOVA ( $<72 \mathrm{~h}$ cohort). Concentrations of hBCATc (A,B) and hBCATm (C,D) proteins were estimated using densitometry analysis and analyzed according to gender and disease state. There was no significant difference observed for hBCATc in either variable, but the concentration of hBCATm in both the frontal and temporal cortex was significant when comparing control males with either the $\mathrm{AD}$ females ( $\mathrm{C}$ and $\mathrm{D}, p=0.004$ and 0.008 , respectively) or $\mathrm{AD}$ males (C and $\mathrm{D}, p=0.008$ and 0.004 , respectively).

\section{S-glutathionylated hBCATm is more evident in $A D$ brain relative to matched controls}

Western blot analysis demonstrated that there was also evidence of differential post-translation modification of hBCATm that was more evident in AD subjects relative to matched controls (Fig. 4I, L (arrow)). Previous work by this group has demonstrated that the $\mathrm{hBCAT}$ proteins have a redox-active CXXC motif that is regulated through changes in the redox environment [32-36]. In particular, these proteins are susceptible to differential S-glutathionylation [35]. Here, we show that the banding pattern observed here reflects S-glutathionylation adducts (Fig. 7), which is more pronounced in $\mathrm{AD}$ brain relative to matched controls.

\section{DISCUSSION}

Glutamate toxicity and necrotic cell death have been implicated in the pathogenesis of ischemia, brain hypoxia, and several neurodegenerative conditions including $\mathrm{AD}$ and amyotrophic lateral sclerosis [2, 3]. This toxicity is considered to be exacerbated by energy deprivation and ischemic conditions, indicating that dysfunction of energy metabolic pathways

\section{Hippocampus}

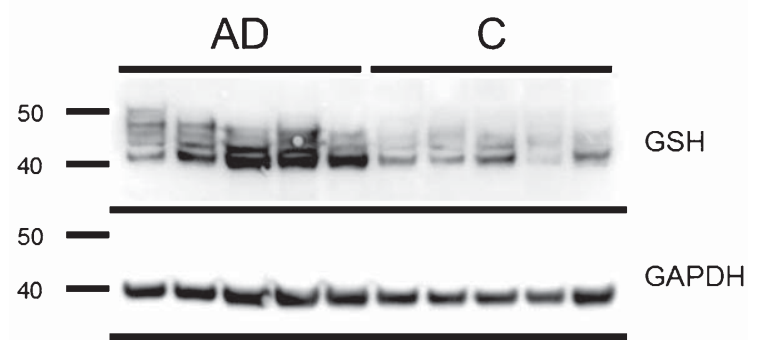

Fig. 7. Western blot analysis of glutathione (GSH) in the hippocampus of $\mathrm{AD}$ and control brains. Western blot analysis was performed as described in the Materials and Methods using antibodies specific to S-glutathionylation (1/1000).

may be responsible [37-39]. Although it is evident that the release of large concentrations of glutamate into the extracellular fluid can occur under pathogenic conditions, the source of this glutamate has been met with extensive debate [9]. However, given that the highest concentration of glutamate is stored in neuronal rather than glial cells, glutamatergic neurons in particular are considered the most likely source. As hBCATc is specifically expressed in glutamatergic and GABAergic neurons and is responsible for catalyzing 
nitrogen shuttling, required for glutamate synthesis it is important to understand if this pathway is dysregulated in neurodegenerative conditions such as $\mathrm{AD}$. In the present study we have shown for the first time that the expression of the hBCAT enzymes, which regulate the concentration of BCAA and glutamate, show a regional increased expression in $\mathrm{AD}$ relative to matched control brains. The upregulation of hBCATm correlated with Braak stage, suggesting that the level of this enzyme is directly related to the progression of the disease. Although studies of cell and animal models suggest that increased concentrations of BCATs may, at least initially, be neuroprotective (see below), BCATs within the brain may increase the production of glutamate and exacerbate neuronal excitotoxicity, potentially contributing to cell death. Altered BCAA metabolism may also contribute to neuronal dysfunction through other mechanisms (e.g., alteration in the concentration of leucine, which regulates several metabolic pathways, such as the mTOR pathway [40]) but the possible contribution of these processes to $\mathrm{AD}$ has yet to be investigated.

Upregulation of hBCATc has previously been reported in rodent models investigating neuronal cell death. Brain-derived neurotrophic factor (BDNF), an important factor in mediating neuronal survival, was shown to prevent degeneration of dorsal lateral geniculate nucleus neurons in newborn rats with visual cortex ablation [41]. In response to BDNF treatment, mRNA fingerprinting showed that BCATc was significantly upregulated, particularly but not exclusively to astrocytes, a curious finding as BCATc is specifically expressed in neuronal cells [42]. In a separate study, the authors demonstrated that upregulation of BCATc mRNA in adult BNDF transgenic mice was particular to the parietal cortex, the hilus, and CA3 hippocampal subfield [43]. This upregulation was restricted to these areas with no reported difference in BCATc expression in the thalamus, the habenula, and the cerebellum. In our study, regional increases in expression were also observed, where increased concentrations of hBCATc showed a significant increase in the hippocampal region of the brain, specifically the CA1 and CA4 region, whereas a significant increase in the frontal or temporal region was not noted. These regional increases support a role for hBCATc in neuronal survival in areas heavily populated with glutamatergic neurons and indicate that BCAT metabolic activity is differentially regulated in these areas.

Our current knowledge of how the glutamate/glutamine cycle is dysregulated in AD may offer insight into why there is an increase in hBCATc expression. Synaptic glutamate excess, which causes neuronal cell death in $\mathrm{AD}$, is partly due to the reduced ability of astrocytes to take up glutamate, because the glutamate transporter (GLT-1/EAAT2) is modified through oxidation [44, 45]. An altered glutamate/glutamine cycle in $\mathrm{AD}$ is also supported by findings of reduced neurotransmitter levels, e.g., glutamate and GABA, in the brains of patients with $\mathrm{AD}$ relative to age-matched controls, whereas the concentration of glutamine was found to be increased [46]. If levels of glutamate recovered from the synapse are reduced, this will compromise the replenishment of glutamate stores in neuronal cells, which may indicate why glutamate levels were found to be low in $\mathrm{AD}$ brain. In our studies, the reported increase in hBCATc expression may be an attempt by the cell to compensate for this lost glutamate, thus acting in a neuroprotective capacity. A neuroprotective role has also been suggested in other models of brain injury, where Kholodilov et al. demonstrated that BCATc was upregulated in the substantia nigra of rat following developmental striatal target injury [47]. In this instance, despite the fact that upregulation correlated with the extent of cell death, a neuroprotective role was suggested because the neurons that were immunopositive for BCATc had normal morphology, with no evidence of apoptotic features. It is possible that in AD too, the upregulation of hBCAT is a physiological response, possibly to oxidative stress (which BCAAs may exacerbate), and in general the neurons that were immunopositive for hBCATc in the human brain tissue also appeared morphologically intact.

Although glutamate is controlled through several metabolic pathways and its levels can be influenced by changes in any of these [48], increased levels of hBCATc would be expected to expand the neuronal pool of glutamate [24, 29, 49]. In this context, as evidenced by the immunohistochemistry data, it may be relevant that the intensity of hBCATc labelling in both cell bodies and dendrites was most pronounced in the hippocampus, a region that is known to be particularly susceptible to excitotoxic neuronal injury [50] and is affected severely and at an early stage of AD. Also, the anti-epileptic drug gabapentin, which inhibits hBCATc [51], has proved successful in treating behavioral alterations in subjects with $\mathrm{AD}[52,53]$. Although hBCATc was not identified as the specific target and further clinical trials would be required to evaluate the utility of gabapentin for treating $\mathrm{AD}$, these reports indicate a potential target for future drug therapies. Altered hBCAT expression or BCAA metabolism has also been reported in several other 
neurodegenerative conditions [54-56]. In addition, to the potential toxicity of glutamate, the $\alpha$-keto acids generated would also create unfavorable conditions for cell survival if these accumulated. The adverse consequences of abnormally increased levels of $\alpha$-keto acids and BCAAs are manifested in the clinical condition maple syrup urine disease [20]. High levels of these metabolites have been associated with inhibition of cell proliferation and a prolonged increase in the G1 stage of the cell division cycle [57]. Neuronal cell models have demonstrated that increased exposure to KIC results in a decrease in cell viability and a loss of cell morphology [58]. Thus, left unregulated, increased levels of hBCATc in neuronal cells of the hippocampus could contribute to a loss in cell viability compromising neuronal function.

In human brain, the mitochondrial isoform is abundant in the endothelial layer of the cerebral vasculature [26] and here shows significant upregulation in AD. Brain glutamate is primarily controlled via the glutamate/glutamine cycle but recently studies supporting the importance of the endothelium in this regulation are gaining impetus [59]. In a study using a bovine endothelial/rat astrocyte coculture model, the $K_{m}$ value of transendothelial glutamate efflux was estimated at $138 \mu \mathrm{M}$ [59]. Under pathophysiological conditions such as ischemia, traumatic head injury, or in some neurodegenerative conditions (including AD), where levels of glutamate are estimated to rise considerably (up to $200 \mu \mathrm{M}$ ), the proposed blood-brain barrier-efflux system could potentially assist in the control of brain glutamate concentrations. Here we propose that when toxicity features, there is increased expression of hBCATm to metabolize this excess glutamate, offering support to astrocytes. However, we have shown that hBCATm levels correlate with Braak stage, and over time sustained overproduction of glutamate and $\alpha$-keto acids may contribute to neuronal dysfunction and damage.

Evidence of post-translational modifications of hBCATm was observed in brain homogenates with increased levels evident in AD brain. The hBCAT proteins are redox sensitive proteins that are susceptible to oxidation, S-nitrosation, and S-glutathionylation, modifications which arise due to changes in the redox environment [32-38]. Changes in cellular stress are intrinsically linked with the pathogenesis of several neurodegenerative diseases including AD. All enzymes of the glutaredoxin system, involved in cellular repair, have been shown to be upregulated in neuronal cells in response to oxidative stress and this correlates with widespread S-glutathionylation
[60]. Here, increased S-glutathionylation of hBCATm occurs in human brains; S-glutathionylation of overexpressed hBCAT in vitro shows evidence of upper bands as the redox environment becomes more oxidizing, which could explain the post-translational modifications seen here. Proteins modified through oxidation and S-nitrosation reported in other studies using AD brains have been shown to be functionally affected, compromising their role in particular under pathogenic conditions, which could also be true for hBCAT $[6,60]$.

In conclusion, this study has clearly demonstrated that the levels of the hBCAT proteins are increased in postmortem AD brain samples, with hBCATm levels correlating with Braak stage indicating a correlation with disease progression and severity. Increased levels of these proteins may initially be part of a neuroprotective cellular response. However, products of their metabolism, glutamate, and the $\alpha$-keto acids are neurotoxic, which may compromise this neuroprotective role, potentially contributing to neuronal cell death. The mechanisms which govern the post-translational modification of the proteins are unclear and require additional investigation. Further studies are needed to ascertain whether reduction of hBCAT levels or activity has the potential to reduce neuronal damage and improve outcome in AD.

\section{ACKNOWLEDGMENTS}

This study was supported by BRACE (Bristol Research into Alzheimer's and Care of the Elderly) awarded to M.E.C. at the University of the West of England. Support for a "Vacation Bursary Studentship" was granted by The Wellcome Trust, UK.

Authors' disclosures available online (http://j-alz. com/manuscript-disclosures/14-2970r1).

\section{SUPPLEMENTARY MATERIAL}

The supplementary material is available in the electronic version of this article: http://dx.doi.org/ 10.3233/JAD-142970.

\section{REFERENCES}

[1] Armstrong R (2006) Plaques and tangles and the pathogenesis of Alzheimer's disease. Folia Neuropathol 44, 1-11.

[2] Choi DW, Maulucci-Gedde M, Kriegstein AR (1987) Glutamate neurotoxicity in cortical cell culture. J Neurosci 7 , 357-368.

[3] Sattler R, Tymianski M (2000) Molecular mechanisms of calcium-dependent excitotoxicity. J Mol Med 78, 3-13. 
[4] Mattson MP, Chan SL (2003) Neuronal and glial calcium signaling in Alzheimer's disease. Cell Calcium 34, 385-397.

[5] Calabrese V, Sultana R, Scapagnini G, Guagliano E, Sapienza M, Bella R, Kanski J, Pennisi G, Mancuso C, Stella AM, Butterfield DA (2006) Nitrosative stress, cellular stress response, and thiol homeostasis in patients with Alzheimer's disease. Antioxid Redox Signal 8, 1975-1986.

[6] Nakamura T, Lipton SA (2007) Molecular mechanisms of nitrosative stress-mediated protein misfolding in neurodegenerative diseases. Cell Mol Life Sci 64, 1609-1620.

[7] Nixon RA (2006) Autophagy in neurodegenerative disease: Friend, foe or turncoat? Trends Neurosci 29, 528-535.

[8] Yang DS, Lee JH, Nixon RA (2009) Monitoring autophagy in Alzheimer's disease and related neurodegenerative diseases. Methods Enzymol 453, 111-144.

[9] Danbolt NC (2001) Glutamate uptake. Prog Neurobiol 65, $1-105$.

[10] Esclaire F, Lesort M, Blanchard C, Hugon J (1997) Glutamate toxicity enhances tau gene expression in neuronal cultures. $J$ Neurosci Res 49, 309-318.

[11] Liang Z, Liu F, Iqbal K, Grundke-Iqbal I, Gong CX (2009) Dysregulation of tau phosphorylation in mouse brain during excitotoxic damage. J Alzheimers Dis 17, 531-539.

[12] Hugon J, Esclaire F, Lesort M, Kisby G, Spencer P (1999) Toxic neuronal apoptosis and modifications of tau and APP gene and protein expressions. Drug Metab Rev 31, 635-647.

[13] Couratier P, Sindou P, Tabaraud F, Diop AG, Spencer PS, Hugon J (1995) Modulation of tau neuronal expression induced by NMDA, non-NMDA and metabotropic glutamate receptor agonists. Neurodegeneration 4, 33-41.

[14] LaNoue KF, Berkich DA, Conway M, Barber AJ, Hu LY, Taylor C, Hutson S (2001) Role of specific aminotransferases in de novo glutamate synthesis and redox shuttling in the retina. J Neurosci Res 66, 914-922.

[15] Ichihara A, Koyama E (1966) Transaminase of branched chain amino acids. $J$ Biochem 59, 160-169

[16] Smith Q, Momma S, Aoyagi M, Rapoport S (1987) Kinetics of neutral amino acid transport across the blood-brain barrier. J Neurochem 49, 1651-1658.

[17] Harris RA, Joshi M, Jeoung NH (2004) Mechanisms responsible for regulation of branched-chain amino acid catabolism. Biochem Biophys Res Commun 313, 391-396.

[18] Chuang DT, Chuang JL, Wynn RM (2006) Lessons from genetic disorders of branched-chain amino acid metabolism. J Nutr 136, 243S-249S.

[19] Chuang JL, Chuang DT (2000) Diagnosis and mutational analysis of maple syrup urine disease using cell cultures. Methods Enzymol 324, 413-423.

[20] Silberman J, Dancis J, Feigin I (1961) Neuropathological observations in maple syrup urine disease: Branched-chain ketoaciduria. Arch Neurol 5, 351-363.

[21] Suryawan A, Hawes JW, Harris RA, Shimomura Y, Jenkins AE, Hutson SM (1998) A molecular model of human branched-chain amino acid metabolism. Am J Clin Nutr $\mathbf{6 8}$, $72-81$.

[22] Than N, Sumegi B, Than G, Bellyei S, Bohn H (2001) Molecular cloning and characterization of placental tissue protein 18(PP18a)/human mitochondrial branched-chain aminotransferase (BCATm) and its novel alternatively spliced PP18b variant. Placenta 22, 235-243.

[23] Lin H, Kaneshige M, Zhao L, Zhang X, Hanover J, Cheng S (2001) An isoform of branched-chain aminotransferase is a novel co-repressor for thyroid hormone nucleur receptors. $J$ Biol Chem 276, 48196-48205.
[24] Garcia-Espinosa MA, Wallin R, Hutson SM, Sweatt AJ (2007) Widespread neuronal expression of branchedchain aminotransferase in the CNS: Implications for leucine/glutamate metabolism and for signaling by amino acids. J Neurochem 100, 1458-1468.

[25] Cole JT, Sweatt AJ, Hutson SM (2012) Expression of mitochondrial branched-chain aminotransferase and $\alpha$-keto-acid dehydrogenase in rat brain: Implications for neurotransmitter metabolism. Front Neuroanat 6, 18.

[26] Hull J, El Hindy M, Kehoe PG, Chalmers K, Love S, Conway ME (2012) Distribution of the branched chain aminotransferase proteins in the human brain and their role in glutamate regulation. J Neurochem $\mathbf{1 2 3}, 997-1009$.

[27] Bixel M, Hutson S, Hamprecht B (1997) Cellular distribution of branched-chain amino acid aminotransferase isoenzymes among rat brain glial cells in culture. J Histochem Cytochem 45, 685-694.

[28] Bixel M, Shimomura Y, Hutson S (2001) Hamprecht B Distribution of key enzymes of branched-chain amino acid metabolism in glial and neuronal cells in culture. J Histochem Cytochem 49, 407-418.

[29] Lieth E, LaNoue KF, Berkich DA, Xu B, Ratz M, Taylor C, Hutson SM (2001) Nitrogen shuttling between neurons and glial cells during glutamate synthesis. J Neurochem $\mathbf{7 6}$, 1712-1723.

[30] Conway ME, Hutson SM (2015) The cytosolic and mitochondrial branched chain aminotransferase. In Branched Chain Amino Acids in Clinical Nutrition. Springer, New York, pp. 25-40.

[31] Davoodi J, Drown PM, Bledsoe RK, Wallin R, Reinhart GD, Hutson SM (1998) Overexpression and characterization of the human mitochondrial and cytosolic branched-chain aminotransferases. J Biol Chem 273, 4982-4989.

[32] Schaffner W, Weissmann C (1973) A rapid, sensitive, and specific method for the determination of protein in dilute solution. Anal Biochem 56, 502-514.

[33] Conway ME, Yennawar N, Wallin R, Poole LB, Hutson SM (2002) Identification of a peroxide-sensitive redox switch at the CXXC motif in the human mitochondrial branched chain aminotransferase. Biochemistry 41, 9070-9078.

[34] Conway ME, Poole LB, Hutson SM (2004) Roles for cysteine residues in the regulatory CXXC motif of human mitochondrial branched chain aminotransferase enzyme. Biochemistry 43, 7356-7364

[35] Yennawar NH, Islam MM, Conway M, Wallin R, Hutson SM (2006) Human mitochondrial branched chain aminotransferase isozyme: Structural role of the CXXC center in catalysis. J Biol Chem 281, 39660-39671.

[36] Conway ME, Coles SJ, Islam MM, Hutson SM (2008) Regulatory control of human cytosolic branched-chain aminotransferase by oxidation and S-glutathionylation and its interactions with redox sensitive neuronal proteins. Biochemistry 47, 5465-5479.

[37] Coles SJ, Easton P, Sharrod H, Hutson SM, Hancock J, Patel VB, Conway ME (2009) S-Nitrosoglutathione inactivation of the mitochondrial and cytosolic BCAT proteins: S-nitrosation and S-thiolation. Biochemistry 48, 645-656.

[38] Shimmyo Y, Kihara T, Akaike A, Niidome T, Sugimoto H (2008) Three distinct neuroprotective functions of myricetin against glutamate-induced neuronal cell death: Involvement of direct inhibition of caspase-3. J Neurosci Res 86, 18361845 .

[39] Haddad GG, Jiang C (1993) O2 deprivation in the central nervous system: On mechanisms of neuronal response, differential sensitivity and injury. Prog Neurobiol 40, 277-318. 
[40] Windelborn JA, Lipton P (2008) Lysosomal release of cathepsins causes ischemic damage in the rat hippocampal slice and depends on NMDA-mediated calcium influx, arachidonic acid metabolism, and free radical production. J Neurochem 106 , 56-69.

[41] McDaniel ML, Marshall CA, Pappan KL, Kwon G (2002) Metabolic and autocrine regulation of the mammalian target of rapamycin by pancreatic beta-cells. Diabetes 51, 28772885.

[42] Caleo M, Menna E, Chierzi S, Cenni MC, Maffei L (2000) Brain-derived neurotrophic factor is an anterograde survival factor in the rat visual system. Curr Biol 10, 1155-1161.

[43] Madeddu F, Naska S, Menna E, Chiellini C, Sweatt AJ, Hutson SM, Benzi L, Maffei M, Maffei L, Bozzi Y (2004) Intraocular delivery of BDNF following visual cortex lesion upregulates cytosolic branched chain aminotransferase (BCATc) in the rat dorsal lateral geniculate nucleus. Eur $J$ Neurosci 20, 580-586.

[44] Castellano S, Macchi F, Scali M, Huang JZ, Bozzi Y (2006) Cytosolic branched chain aminotransferase (BCATc) mRNA is up-regulated in restricted brain areas of BDNF transgenic mice. Brain Res 1108, 12-18.

[45] Mattson MP, Guo ZH, Geiger JD (1999) Secreted form of amyloid precursor protein enhances basal glucose and glutamate transport and protects against oxidative impairment of glucose and glutamate transport in synaptosomes by a cyclic GMP-mediated mechanism. J Neurochem 73, 532-537.

[46] Beart PM, O'Shea RD (2007) Transporters for L-glutamate: An update on their molecular pharmacology and pathological involvement. Br J Pharmacol 150, 5-17.

[47] Gueli MC, Taibi G (2013) Alzheimer's disease: Amino acid levels and brain metabolic status. Neurol Sci 34, 1575-1579.

[48] Kholodilov N, Neystat M, Oo T, Hutson S, Burke R (2000) Upregulation of cytosolic branched chain aminotransferase in substantia nigra following developmental striatal target injury. Mol Brain Res 75, 281-286.

[49] McKenna MC (2007) The glutamate-glutamine cycle is not stoichiometric: Fates of glutamate in brain. $J$ Neurosci Res 85, 3347-3358.

[50] Sweatt A, Wood M, Suryawan A, Wallin R, Willingham M, Hutson S (2004) Branched-chain amino acid catabolism: Unique segregation of pathway enzymes in organ systems and peripheral nerves. Am J Physiol Endocrinol Metab 286, E64-E76.
[51] Mufson EJ, Binder L, Counts SE, DeKosky ST, de ToledoMorrell L, Ginsberg SD, Ikonomovic MD, Perez SE, Scheff SW (2012) Mild cognitive impairment: Pathology and mechanisms. Acta Neuropathol 123, 13-30.

[52] Goto M, Miyahara I, Hirotsu K, Conway M, Yennawar N, Islam MM, Hutson SM (2005) Structural determinants for branched-chain aminotransferase isozyme-specific inhibition by the anticonvulsant drug Gabapentin. J Biol Chem 280, 37246-37256.

[53] Moretti R, Torre P, Antonello RM, Cazzato G, Bava A (2003) Gabapentin for the treatment of behavioural alterations in dementia: Preliminary 15-month investigation. Drugs Aging 20, 1035-1040.

[54] Kim Y, Wilkins KM, Tampi RR (2008) Use of gabapentin in the treatment of behavioural and psychological symptoms of dementia: A review of the evidence. Drugs Aging 25, 187-196.

[55] Bossers K, Meerhoff G, Balesar R, Van Dongen JW, Kruse CG, Swaab DF, Verhaagen J (2009) Analysis of gene expression in Parkinson's disease: Possible involvement of neurotrophic support and axon guidance in dopaminergic cell death. Brain Pathol 19, 91-107.

[56] Uchida Y, Ohtsuki S, Katsukura Y, Ikeda C, Suzuki T, Kamiie J, Terasaki T (2011) Quantitative targeted absolute proteomics of human blood-brain barrier transporters and receptors. $J$ Neurochem 117, 333-345.

[57] Mochel F, Benaich S, Rabier D, Durr A (2011) Validation of plasma branched chain amino acids as biomarkers in Huntington disease. Arch Neurol 68, 265-267.

[58] Liao CL, Herman MM, Bensch KG (1978) Prolongation of G1 and $\mathrm{S}$ phase in C-6 glioma cells treated with maple syrup urine disease metabolits. Morphologic and Cell Cycle Studies. Lab Invest 38, 122-133.

[59] Fernstrom J (2005) Branched-chain amino acids and brain function. J Nutr 135, 1539S-1546S.

[60] Helms HC, Madelung R, Waagepetersen HS, Nielsen CU, Brodin B (2012) In vitro evidence for the brain glutamate efflux hypothesis: Brain endothelial cells cocultured with astrocytes display a polarized brain-to-blood transport of glutamate. Glia 60, 882-893.

[61] Butterfield A, Faipoon H, Clair D, Keller JN, Pierce WM, Klein JB, Markesbery WR (2006) Redox proteomics identification of oxidatively modified hippocampal proteins in mild cognitive impairment: Insights into the development of Alzheimer's disease. Neurobiol Dis 22, 223-232. 